\title{
On solving an $n \times n$ system of nonlinear Volterra integral equations by the Newton-Kantorovich method
}

\author{
Hameed Husam Hameed $^{\mathrm{a}, \mathrm{d}}$, Z.K. Eshkuvatov ${ }^{\mathrm{b}, \mathrm{c}, *}$, N.M.A. Nik Long ${ }^{\mathrm{a}, \mathrm{c}}$ \\ a Department of Mathematics, Faculty of Science, Universiti Putra Malaysia (UPM), Selangor, Malaysia \\ b Faculty of Science and Technology, Universiti Sains Islam Malaysia (USIM), Negeri Sembilan, Malaysia \\ c Institute for Mathematical Research, Universiti Putra Malaysia (UPM), Selangor, Malaysia \\ d Technical Institute of Alsuwerah, the Middle Technical University, Iraq
}

*Corresponding author, e-mail: zainidin@usim.edu.my

Received 31 Aug 2014

Accepted 20 Jul 2016

\begin{abstract}
We consider an $n \times n$ system of nonlinear integral equations of Volterra type (nonlinear VIEs) arising from an economic model. By applying the Newton-Kantorovich method to the nonlinear VIEs we linearize them into linear Volterra type integral equations (linear VIEs). Uniqueness of the solution of the system is shown. An idea has been proposed to find the approximate solution by transforming the system of linear VIEs into a system of linear Fredholm integral equations by using sub-collocation points. Then the backward Newton interpolation formula is used to find the approximate solution at the collocation points. Each iteration is solved by the Nystrom type Gauss-Legendre quadrature formula (QF). It is found that by increasing the number of collocation points of QF with fewer iterations, a high accurate approximate solution can be obtained. Finally, an illustrative example is demonstrated to validate the accuracy of the method.
\end{abstract}

KEYWORDS: nonlinear integral operator, Volterra integral type, Gauss-Legendre method

MSC2010: 45G15

\section{INTRODUCTION}

The Newton-Kantorovich method is a powerful technique for solving nonlinear problems, and the Kantorovich theorems are significant in nonlinear analysis to prove the existence and uniqueness of the solution of nonlinear integral equations arising in many scientific areas. In recent years, many different methods have been used to obtain the solution of the system of integral equations, such as Adomian decomposition method ${ }^{1}$, step method $^{2}$, modified reproducing kernel method ${ }^{3}$, Chebyshev wavelets method ${ }^{4}$, biorthogonal systems ${ }^{5}$ and series method ${ }^{6}$. In this note, we consider the system of nonlinear Volterra integral equations

$$
\begin{aligned}
x_{i}(t) & =\sum_{\kappa=1}^{r} \int_{y(t)}^{t} H_{i k}(t, \tau) \Psi\left(x_{\kappa}(\tau)\right) \mathrm{d} \tau, \\
z_{j}(t) & =\sum_{\kappa=1}^{r} \int_{y(t)}^{t} K_{j \kappa}(t, \tau) \Psi\left(x_{\kappa}(\tau)\right) \mathrm{d} \tau, \\
c(t) & =\sum_{i=1}^{r} x_{i}(t)+\sum_{j=1}^{p} z_{j}(t),
\end{aligned}
$$

where $i=1,2, \ldots, r, j=1,2, \ldots, p, r+p+1=n$ and $\Psi(u)=u^{m}, m \geqslant 2$ is an integer. The unknown functions are

$$
\begin{aligned}
x_{i}(t) \in C\left[t_{0}, T\right], & i=1,2, \ldots, r, \\
z_{j}(t) \in C\left[t_{0}, T\right], & j=1,2, \ldots, p, \\
y(t) \in C^{1}\left[t_{0}, T\right], &
\end{aligned}
$$

where $0<t_{0} \leqslant t \leqslant T, y(t)<t, y(t) \geqslant y\left(t_{0}\right)=t_{0}>$ 0 , and the kernel functions $H_{i \kappa}(t, \tau), K_{j k}(t, \tau) \in$ $C\left(\left[t_{0}, T\right] \times\left[t_{0}, T\right]\right), i, \kappa=1,2, \ldots, r ; j=1,2, \ldots, p$ and non-negative. The system (1) represents the $n$ commodity model where $x_{i}(t), i=1,2, \ldots, r$ is the reconstruction rate of $i$ th commodity of kind $I$ used for performing internal function of the system, and $z_{j}(t), j=1,2, \ldots, p$ is the reconstruction rate of $j$ th commodity of kind $I I$ used for performing external functions of the system, and $H_{i \kappa}(t, \tau), K_{j \kappa}(t, \tau)$, $i, \kappa=1,2, \ldots, r ; j=1,2, \ldots, p$, are the productivities of the generation of the $i$ th commodity of kind $I$ and the $j$ th commodity of kind $I I$ with the use of the corresponding $\kappa$ th commodity. The function $y(t)$ corresponding to the intensity of using commodities of kind $I$ at time $t$. Boikov and Tynda ${ }^{7}$ implemented 
the Newton-Kantorovich method to solve (1) when $\Psi(u)=u$ with the same conditions above for the known and unknown functions.

The paper is arranged as follows. We describe the Newton-Kantorovich method. Then we solve the system of linear integral equation of Volterra type using Nystrom type Gauss-Legendre quadrature formula. An example is described to show the accuracy and efficiency of the method. The last section concludes the main ideas of the approximate method.

\section{DESCRIPTION OF THE METHOD}

In solving (1) let

$$
\begin{aligned}
P_{i}(V) & =x_{i}(t)-\sum_{\kappa=1}^{r} \int_{y(t)}^{t} H_{i \kappa}(t, \tau) \Psi\left(x_{\kappa}(\tau)\right) \mathrm{d} \tau=0 \\
P_{r+j}(V) & =z_{j}(t)-\sum_{\kappa=1}^{r} \int_{y(t)}^{t} K_{j \kappa}(t, \tau) \Psi\left(x_{\kappa}(\tau)\right) \mathrm{d} \tau=0 \\
P_{n}(V) & =c(t)-\sum_{i=1}^{r} x_{i}(t)-\sum_{j=1}^{p} z_{j}(t)=0
\end{aligned}
$$

where $i=1,2, \ldots r, j=1,2, \ldots p, V=(X, Z, y)$, $r+p+1=n, X=\left(x_{1}(t), x_{2}(t), \ldots, x_{r}(t)\right), Z=$ $\left(z_{1}(t), z_{2}(t), \ldots, z_{p}(t)\right)$, then the system of (1) can be reduced to the operator form

$$
\begin{gathered}
P(V)=\left(P_{i}(V), P_{r+j}(V), P_{n}(V)\right)=(0,0,0), \\
i=1, \ldots, r, \quad j=1, \ldots, p, \quad n=r+p+1 .
\end{gathered}
$$

Eq. (3) can be solved by Newton-Kantorovich method. Let the initial approximation be

$$
P^{\prime}\left(V_{0}\right)\left(V-V_{0}\right)+P\left(V_{0}\right)=0,
$$

where $V_{0}=\left(X_{0} ; Z_{0} ; y_{0}\right)=\left(x_{10}(t), \ldots, x_{r 0}(t) ; z_{10}(t)\right.$, $\left.\ldots, z_{p 0}(t) ; y_{0}(t)\right)$ denotes the initial guess and can be chosen as any continuous functions provided that $y_{0}(t)<t$. The Fréchet derivative of $P(V)$ at the point $V_{0}$ is defined as

$$
\begin{gathered}
P^{\prime}\left(V_{0}\right) V=\left(\lim _{s \rightarrow 0} \frac{1}{s}\left[P_{i}\left(V_{0}+s V\right)-P_{i}\left(V_{0}\right)\right],\right. \\
\lim _{s \rightarrow 0} \frac{1}{s}\left[P_{r+j}\left(V_{0}+s V\right)-P_{r+j}\left(V_{0}\right)\right], \\
\left.\lim _{s \rightarrow 0} \frac{1}{s}\left[P_{n}\left(V_{0}+s V\right)-P_{n}\left(V_{0}\right)\right]\right), \\
i=1,2, \ldots, r, \quad j=1,2, \ldots, p, \quad n=r+p+1,
\end{gathered}
$$

since $V_{0}=\left(X_{0} ; Z_{0} ; y_{0}\right)$ and $V=(X ; Z ; y)$. Then we obtain $P^{\prime}\left(V_{0}\right) V=$

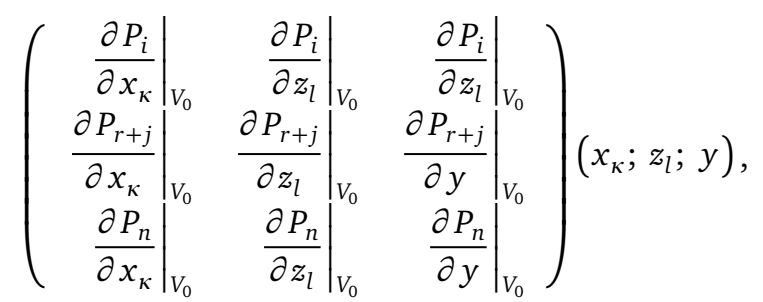

where $\kappa=1,2, \ldots, r ; l=1,2, \ldots, p$. Hence (5) represents the Fréchet derivative of nonlinear operator $P(V)$ at the point $V_{0}$, where

$$
\begin{aligned}
& \left.\frac{\partial P_{i}(V)}{\partial x_{\kappa}}\right|_{V_{0}}=\delta_{i \kappa} x_{\kappa}(t) \\
& -m \int_{y_{0}(t)}^{t} H_{i \kappa}(t, \tau) x_{\kappa}^{m-1}(\tau) x_{\kappa}(\tau) \mathrm{d} \tau \\
& i, \kappa=1,2, \ldots, r \text {, } \\
& \left.\frac{\partial P_{i}(V)}{\partial z_{l}}\right|_{V_{0}}=0, \quad i=1, \ldots, r, \quad l=1, \ldots, p, \\
& \left.\frac{\partial P_{i}(V)}{\partial y}\right|_{V_{0}}=\sum_{\kappa=1}^{r} H_{i \kappa}\left(t, y_{0}(t)\right) x_{\kappa 0}^{m}\left(y_{0}(t)\right) y(t) \text {, } \\
& i=1,2, \ldots, r, \\
& \left.\frac{\partial P_{r+j}(V)}{\partial x_{\kappa}}\right|_{V_{0}}=-m \int_{y_{0}(t)}^{t} K_{j \kappa}(t, \tau) x_{\kappa 0}^{m-1}(\tau) x_{\kappa}(\tau) \mathrm{d} \tau \text {, } \\
& j=1, \ldots, p, \kappa=1, \ldots, r, \\
& \left.\frac{\partial P_{r+j}(V)}{\partial z_{l}}\right|_{V_{0}}=\delta_{j l} z_{l}, \quad j, l=1, \ldots, p, \\
& \left.\frac{\partial P_{r+j}(V)}{\partial y}\right|_{V_{0}}=\sum_{\kappa=1}^{r} K_{j \kappa}\left(t, y_{0}(t)\right) x_{\kappa 0}^{m}\left(y_{0}(t)\right) y(t), \\
& j=1,2, \ldots, p, \\
& \left.\frac{\partial P_{n}(V)}{\partial x_{\kappa}}\right|_{V_{0}}=-x_{\kappa}(t), \quad \kappa=1, \ldots, r \\
& \left.\frac{\partial P_{n}(V)}{\partial z_{l}}\right|_{V_{0}}=-z_{l}(t), \quad l=1, \ldots, p, \\
& \left.\frac{\partial P_{n}(V)}{\partial y}\right|_{V_{0}}=0 \text {, }
\end{aligned}
$$

where $\delta_{i \kappa}$ denotes the Kronecker delta. Thus (4) has the form

$$
\begin{gathered}
\Delta x_{i}(t)-m \sum_{\kappa=1}^{r} \int_{y_{0}(t)}^{t} H_{i \kappa}(t \tau) x_{\kappa 0}^{m-1}(\tau) \Delta x_{\kappa}(\tau) \mathrm{d} \tau \\
+\Delta y(t) \sum_{\kappa=1}^{r} H_{i \kappa}\left(t, y_{0}(t)\right) x_{\kappa 0}^{m}\left(y_{0}(t)\right)
\end{gathered}
$$




$$
\begin{aligned}
& =\sum_{\kappa=1}^{r} \int_{y_{0}(t)}^{t} H_{i \kappa}(t, \tau) x_{\kappa 0}^{m}(\tau)-x_{i 0}(t) \\
& \Delta z_{j}(t)-m \sum_{\kappa=1}^{r} \int_{y_{0}(t)}^{t} K_{j \kappa}(t, \tau) x_{\kappa 0}^{m-1}(\tau) \Delta x_{\kappa}(\tau) \mathrm{d} \tau \\
& \quad+\Delta y(t) \sum_{\kappa=1}^{r} K_{j \kappa}\left(t, y_{0}(t)\right) x_{\kappa 0}^{m}\left(y_{0}(t)\right) \\
& =\sum_{\kappa=1}^{r} \int_{y_{0}(t)}^{t} K_{j \kappa}(t, \tau) x_{\kappa 0}^{m}(\tau) \mathrm{d} \tau-z_{j 0}(t) \\
& \sum_{i=1}^{r} \Delta x_{i}(t)+\sum_{j=1}^{p} \Delta z_{j}(t) \\
& =c(t)-\sum_{i=1}^{r} x_{i 0}(t)-\sum_{j=1}^{p} z_{j 0}(t)
\end{aligned}
$$

where

$$
\begin{array}{ll}
\Delta x_{i}(t)=x_{i 1}(t)-x_{i 0}(t), & i=1,2, \ldots, r, \\
\Delta z_{j}(t)=j_{j 1}(t)-x_{j 0}(t), & j=1,2, \ldots, p, \\
\Delta y(t)=y_{1}(t)-y_{0}(t), &
\end{array}
$$

and $\left(x_{10}(t), \ldots, x_{r 0}(t) ; z_{10}(t), \ldots, z_{p 0}(t) ; y_{0}(t)\right)$ is the initial approximation. By solving the system (6) for $\Delta x_{i}, i=1, \ldots, r ; \Delta z_{j}, j=1, \ldots, p$; and $\Delta y$ we obtain $\left(x_{11}(t), \ldots, x_{r 1}(t) ; z_{11}(t), \ldots, z_{p 1}(t) ; y_{1}(t)\right)$. In the same manner, a sequence of approximate solutions $V_{q}(t)=\left(X_{q}(t), y_{q}, Z_{q}\right)=\left(x_{i(q)}, y_{q}, z_{j(q)}\right)$, $q=2,3, \ldots$ can be assessed from the equation

$$
P^{\prime}\left(V_{0}\right)\left(V_{q}-V_{q-1}\right)+P\left(V_{q-1}\right)=0, \quad q=2,3, \ldots
$$

which is equivalent to the system

$$
\begin{aligned}
& \Delta x_{i(q)}(t) \\
& \quad-m \sum_{\kappa=1}^{r} \int_{y_{0}(t)}^{t} H_{i \kappa}(t \tau) x_{\kappa 0}^{m-1}(\tau) \Delta x_{\kappa(q)}(\tau) \mathrm{d} \tau \\
& \quad+\Delta y_{q}(t) \sum_{\kappa=1}^{r} H_{i \kappa}\left(t, y_{0}(t)\right) x_{\kappa 0}^{m}\left(y_{0}(t)\right) \\
& =\sum_{\kappa=1}^{r} \int_{y_{0}(t)}^{t} H_{i \kappa}(t, \tau) x_{\kappa 0}^{m}(\tau)-x_{i 0}(t), \\
& \Delta z_{j(q)}(t) \\
& \quad-m \sum_{\kappa=1}^{r} \int_{y_{0}(t)}^{t} K_{j \kappa}(t, \tau) x_{\kappa 0}^{m-1}(\tau) \Delta x_{\kappa(q)}(\tau) \mathrm{d} \tau \\
& \quad+\Delta y_{q}(t) \sum_{\kappa=1}^{r} K_{j \kappa}\left(t, y_{0}(t)\right) x_{\kappa 0}^{m}\left(y_{0}(t)\right) \\
& =\sum_{\kappa=1}^{r} \int_{y_{0}(t)}^{t} K_{j \kappa}(t, \tau) x_{\kappa 0}^{m}(\tau) \mathrm{d} \tau-z_{j 0}(t),
\end{aligned}
$$

$$
\begin{aligned}
& \sum_{i=1}^{r} \Delta x_{i(q)}(t)+\sum_{j=1}^{p} \Delta z_{j(q)}(t) \\
& =c(t)-\sum_{i=1}^{r} x_{i 0}(t)-\sum_{j=1}^{p} z_{j 0}(t),
\end{aligned}
$$

where

$$
\begin{aligned}
\Delta x_{i(q)}(t) & =x_{i(q)}(t)-x_{i(q-1)}(t), & & i=1, \ldots, r, \\
\Delta z_{j(q)}(t) & =j_{j(q)}(t)-x_{j(q-1)}(t), & & j=1, \ldots, p, \\
\Delta y_{q}(t) & =y_{q}(t)-y_{q-1}(t), & & q=2,3, \ldots .
\end{aligned}
$$

Solving the system (7) for $\Delta x_{i(q)}(t), \Delta y_{q}(t)$, $\Delta z_{j(q)}(t)$ yields sequences of approximate solutions $x_{i(q)}(t), y_{q}(t)$, and $z_{j(q)}(t)$, where $i=1,2, \ldots r, j=$ $1,2, \ldots p$, and $q=2,3, \ldots$.

Lemma 1 If the system of (6) has a unique solution $V^{*}=\left(X^{*}, y^{*}, Z^{*}\right)=\left(x_{i}^{*}, y^{*}, z_{j}^{*}\right), i=1,2, \ldots r, j=$ $1,2, \ldots p,\|\Delta V\| \leqslant \eta$ and $\left\|P^{\prime \prime}(V)\right\| \leqslant \zeta$, where $\eta$ and $\zeta$ are the coefficients of Kantorovich's majorant function, $R(t)=\zeta t^{2}-2 t+2 \eta$. Then the sequence of successive approximation $V_{q}(t)=\left(X_{q}(t), y_{q}(t), Z_{q}(t)\right)=$ $\left(x_{i(q)}(t), y_{q}(t), z_{j(q)}(t)\right), q=2,3, \ldots$ converges to the solution $V^{*}(t)=\left(X^{*}(t), y^{*}(t), Z^{*}(t)\right)$ and the rate of convergence is

$$
\left\|V^{*}-V_{q}\right\| \leqslant \frac{1}{\zeta}(1-\sqrt{1-2 \zeta \eta})^{q+1}, \quad q=1,2, \ldots .
$$

Proof: We set

$$
\begin{aligned}
& L_{i}^{1}(t)=\sum_{\kappa=1}^{r} H_{i \kappa}\left(t, y_{0}(t)\right) x_{\kappa 0}^{m}\left(y_{0}(t)\right), \\
& L_{j}^{2}(t)=\sum_{\kappa=1}^{r} K_{j \kappa}\left(t, y_{0}(t)\right) x_{\kappa 0}^{m}\left(y_{0}(t)\right), \\
& F_{i}^{1}(t)=\int_{y_{0}(t)}^{t} \sum_{\kappa=1}^{r} H_{i \kappa}(t, \tau) x_{\kappa 0}^{m}(\tau) \mathrm{d} \tau-x_{i 0}(t), \\
& F_{j}^{2}(t)=\int_{y_{0}(t)}^{t} \sum_{\kappa=1}^{r} K_{j \kappa}(t, \tau) x_{\kappa 0}^{m}(\tau) \mathrm{d} \tau-z_{i 0}(t), \\
& F^{3}(t)=c(t)-\sum_{i=1}^{r} x_{i 0}(t)-\sum_{j=1}^{p} z_{j 0}(t),
\end{aligned}
$$

where $i=1, \ldots, r$ and $j=1, \ldots, p$. As consequently, the system (6) can be represented as

$$
\begin{gathered}
\Delta x_{i}(t)-m \sum_{\kappa=1}^{r} \int_{y_{0}(t)}^{t} H_{i \kappa}(t, \tau) x_{\kappa 0}^{m-1}(\tau) \Delta x_{\kappa}(\tau) \mathrm{d} \tau \\
+\Delta y(t) L_{i}^{1}(t)=F_{i}^{1}(t), \quad i=1, \ldots, r
\end{gathered}
$$




$$
\begin{aligned}
& \Delta z_{j}(t)-m \sum_{\kappa=1}^{r} \int_{y_{0}(t)}^{t} K_{j \kappa}(t, \tau) x_{\kappa 0}^{m-1}(\tau) \Delta x_{\kappa}(\tau) \mathrm{d} \tau \\
& \quad+\Delta y(t) L_{j}^{2}(t)=F_{j}^{2}(t), \quad j=1, \ldots, p \\
& \sum_{i=1}^{r} \Delta x_{i}(t)+\sum_{j=1}^{p} \Delta z_{j}(t)=F^{3}(t)
\end{aligned}
$$

By expressing $\Delta y(t)$ from the first and second equations of the system (8), we obtain

$$
\begin{array}{r}
\Delta y L_{i}^{1}(t)=F_{i}^{1}(t)-\Delta x_{i}(t) \\
+m \sum_{\kappa=1}^{r} \int_{y_{0}(t)}^{t} H_{i \kappa}(t \tau) x_{\kappa 0}^{m-1}(\tau) \Delta x_{\kappa}(\tau) \mathrm{d} \tau \\
i=1, \ldots, r
\end{array}
$$

and

$$
\begin{aligned}
& \Delta y L_{j}^{2}(t)=F_{j}^{2}(t)-\Delta z_{j}(t) \\
& +m \sum_{\kappa=1}^{r} \int_{y_{0}(t)}^{r} K_{j \kappa}(t, \tau) x_{\kappa 0}^{m-1}(\tau) \Delta x_{\kappa}(\tau) \mathrm{d} \tau, \\
& \quad j=1, \ldots, p .
\end{aligned}
$$

We add the first $r$ equations of (9) to obtain

$$
\begin{array}{r}
\Delta y(t) \sum_{i=1}^{r} L_{i}^{1}(t)=\sum_{i=1}^{r} F_{i}^{1}(t)-\sum_{i=1}^{r} \Delta x_{i}(t) \\
+m \sum_{i=1}^{r} \sum_{k=1}^{r}\left[\int_{y_{0}(t)}^{t} H_{i \kappa}(t, \tau) x_{\kappa 0}^{m-1}(\tau)\right. \\
\left.\Delta x_{\kappa}(\tau)\right] \mathrm{d} \tau,
\end{array}
$$

and we do summation of equations in (10) with respect to $r+j, j=1,2, \ldots, p$ to obtain

$$
\begin{array}{r}
\Delta y(t) \sum_{i=1}^{p} L_{j}^{2}(t)=\sum_{j=1}^{p} F_{j}^{2}(t)-\sum_{j=1}^{p} \Delta z_{j}(t) \\
+m \sum_{j=1}^{p} \sum_{k=1}^{r} \int_{y_{0}(t)}^{t}\left[K_{j \kappa}(t, \tau) x_{\kappa 0}^{m-1}(\tau)\right. \\
\left.\Delta x_{\kappa}(\tau)\right] \mathrm{d} \tau .
\end{array}
$$

Then, by adding (11) and (12), we obtain

$$
\Delta y(t)\left[\sum_{i=1}^{r} L_{i}^{1}(t)+\sum_{j=1}^{p} L_{j}^{2}(t)\right]
$$

$$
\begin{gathered}
=\sum_{i=1}^{r} F_{i}^{1}(t)+\sum_{i=1}^{r} F_{i}^{2}(t)-F^{3}(t) \\
+m \sum_{i=1}^{r} \int_{y_{0}(t)}^{t}\left[\sum_{\kappa=1}^{r} H_{\kappa i}(t, \tau) x_{i 0}^{m-1}(\tau) \Delta x_{i}(\tau)\right. \\
\left.+\sum_{\kappa=1}^{p} K_{\kappa i}(t, \tau) x_{i 0}^{m-1}(\tau) \Delta x_{i}(\tau)\right] \mathrm{d} \tau .
\end{gathered}
$$

Assume that $G(t)=\left[\sum_{i=1}^{r} F_{i}^{1}(t)+\sum_{i=1}^{p} F_{i}^{2}(t)\right]$ has no zeros on the interval $\left[t_{0}, T\right]$. Then $\Delta y(t)$ has the form

$$
\begin{aligned}
\Delta y(t)=\frac{1}{G(t)} & {\left[m \sum _ { i = 1 } ^ { r } \int _ { y _ { 0 } ( t ) } ^ { t } \left[\sum _ { \kappa = 1 } ^ { r } \left(H_{\kappa i}(t, \tau) x_{i 0}^{m-1}(\tau)\right.\right.\right.} \\
\left.\Delta x_{i}(\tau)\right) & \left.+\sum_{\kappa=1}^{p} K_{\kappa i}(t, \tau) x_{i 0}^{m-1}(\tau) \Delta x_{i}(\tau)\right] \mathrm{d} \tau \\
& \left.+\sum_{i=1}^{r} F_{i}^{1}(t)+\sum_{j=1}^{p} F_{j}^{2}(t)-F^{3}(t)\right] .
\end{aligned}
$$

To find $\Delta x_{i}(t), i=1,2, \ldots, r$ we substitute (14) in the first equation of the system (8)

$$
\begin{array}{r}
\Delta x_{i}(t)-m \sum_{j=1}^{r} \int_{y_{0}(t)}^{t} H_{i j}(t, \tau) x_{j 0}^{m-1}(\tau) \Delta x_{j}(\tau) \mathrm{d} \tau \\
+\frac{L_{i}^{1}}{G(t)}\left[m \sum _ { j = 1 } ^ { r } \int _ { y _ { 0 } ( t ) } ^ { t } \left[\sum _ { \kappa = 1 } ^ { r } \left(H_{\kappa j}(t, \tau) x_{j 0}^{m-1}(\tau)\right.\right.\right. \\
\left.\left.\Delta x_{j}(\tau)\right)+\sum_{\kappa=1}^{p} K_{\kappa j}(t, \tau) x_{j 0}^{m-1}(\tau) \Delta x_{j}(\tau)\right] \mathrm{d} \tau \\
\left.+\sum_{i=1}^{r} F_{i}^{1}(t)+\sum_{j=1}^{p} F_{j}^{2}(t)-F^{3}(t)\right]=F_{i}^{1}(t),
\end{array}
$$

then

$$
\begin{aligned}
\Delta x_{i}(t)+m \sum_{j=1}^{r} \int_{y_{0}(t)}^{t}\left(\Omega_{i j}(t, \tau) x_{j 0}^{m-1}(\tau)\right. \\
\left.\Delta x_{j}(\tau) \mathrm{d} \tau\right)=\Phi_{i}(t), \quad i=1, \ldots, r,
\end{aligned}
$$

where

$$
\begin{aligned}
\Omega_{i j}(t, \tau)= & \frac{L_{i}^{1}(t)}{G(t)}\left[\sum_{\kappa=1}^{r} H_{\kappa j}(t, \tau)\right. \\
& \left.+\sum_{\kappa=1}^{p} K_{\kappa j}(t, \tau)\right]-H_{i j}(t, \tau) \\
\Phi_{i}(t)= & F_{i}^{1}(t)+\frac{L_{i}^{1}(t)}{G(t)}\left[F^{3}(t)\right. \\
& \left.-\sum_{i=1}^{r} F_{i}^{1}(t)+\sum_{j=1}^{p} F_{j}^{2}(t)\right] .
\end{aligned}
$$


$\Delta z_{i}(t)$ can be evaluated from the second equation of the system (8) to be of the form

$$
\begin{gathered}
\Delta z_{j}(t)=F_{j}^{2}(t)+m \sum_{\kappa=1}^{r} \int_{y_{0}(t)}^{t}\left(K_{j \kappa}(t, \tau) x_{\kappa 0}^{m-1}(\tau)\right. \\
\left.\Delta x_{\kappa}(\tau)\right) \mathrm{d} \tau-\Delta y(t) L_{j}^{2}(t), j=1, \ldots, p .
\end{gathered}
$$

Eq. (16) represents a standard system of linear system of Volterra integral equation of the second kind with respect to $\Delta x_{i}(t)$, and from the theory of integral equations it has a unique solution provided that $\Omega_{i j}(t, \tau)$ and $\Phi_{i}(t)$ are continuous functions in their range of definitions and $G(t)$ has no zeros on $\left[t_{0}, T\right]$. Then by using the concept of Kantorovich's Theorem $^{8}$, we can reveal that $V^{*}(t)$ is the unique solution to the operator system (2) and $\left\|V^{*}-V_{q}\right\| \leqslant$ $(1 / \zeta)(1-\sqrt{1-2 \zeta \eta})^{q+1}, q=1,2, \ldots$

By solving (14), (16) and () in terms of $\Delta y(t)$, $\Delta x_{i}(t), i=1,2, \ldots, r$ and $\Delta y_{j}(t), j=1,2, \ldots, p$ we obtain $\left(x_{i 1}(t), z_{1 j}(t), y_{1}(t)\right)$, and for the process of successive approximation we have

$$
\begin{aligned}
& \Delta x_{i(q)}(t)+m \sum_{j=1}^{r} \int_{y_{0}(t)}^{t}\left(\Omega_{i j}(t, \tau) x_{j 0}^{m-1}(\tau)\right. \\
& \left.\Delta x_{j(q)}(\tau)\right) \mathrm{d} \tau=\Phi_{i(q-1)}(t), \quad i=1, \ldots, r, \\
& \Delta y_{q}(t)=\frac{1}{G(t)}\left[m \sum _ { i = 1 } ^ { r } \int _ { y _ { 0 } ( t ) } ^ { t } \left[\sum _ { \kappa = 1 } ^ { r } \left(H_{\kappa i}(t, \tau)\right.\right.\right. \\
& \left.x_{i 0}^{m-1}(\tau) \Delta x_{i(q)}(\tau)\right) \\
& \left.+\sum_{\kappa=1}^{p} K_{\kappa i}(t, \tau) x_{i 0}^{m-1}(\tau) \Delta x_{i(q)}(\tau)\right] \mathrm{d} \tau \\
& \left.+\sum_{i=1}^{r} F_{i(q-1)}^{1}(t)+\sum_{j=1}^{p} F_{j(q-1)}^{2}(t)-F_{q-1}^{3}(t)\right], \\
& \Delta z_{j(q)}(t)=F_{j(q-1)}^{2}(t) \\
& +m \sum_{\kappa=1}^{r} \int_{y_{0}(t)}^{t} K_{j \kappa}(t, \tau) x_{\kappa 0}^{m-1}(\tau) \Delta x_{\kappa(q)}(\tau) \mathrm{d} \tau \\
& \quad+\Delta y(t)_{q} L_{j(q-1)}^{2}(t), \quad j=1, \ldots, p, \quad
\end{aligned}
$$

where $q=2,3, \ldots$, and

$$
\begin{aligned}
\Phi_{i(q-1)}(t)= & F_{i(q-1)}^{1}(t)+\frac{L_{i(q-1)}^{1}(t)}{G(t)}\left[F_{s-1}^{3}(t)\right. \\
& \left.-\sum_{i=1}^{r} F_{i(q-1)}^{1}(t)+\sum_{j=1}^{p} F_{j(q-1)}^{2}(t)\right], \\
F_{i(q-1)}^{1}(t)= & \int_{y_{0}(t)}^{t} \sum_{\kappa=1}^{r} H_{i \kappa}(t, \tau) x_{\kappa(q-1)}^{m}(\tau) \mathrm{d} \tau \\
& -x_{i(q-1)}(t), \quad i=1, \ldots, r,
\end{aligned}
$$

$$
\begin{aligned}
F_{j(q-1)}^{2}(t)= & \int_{y_{0}(t)}^{t} \sum_{\kappa=1}^{r} K_{j \kappa}(t, \tau) x_{\kappa(q-1)}^{m}(\tau) \mathrm{d} \tau \\
& -z_{i(q-1)}(t), \quad j=1, \ldots, p, \\
F_{q-1}^{3}(t)= & c(t)-\sum_{i=1}^{r} x_{i(q-1)}(t)-\sum_{j=1}^{p} z_{j(q-1)}(t), \\
L_{i(q-1)}^{1}(t)= & \sum_{\kappa=1}^{r} H_{i \kappa}\left(t, y_{q-1}(t)\right) x_{\kappa(q-1)}^{m}\left(y_{q-1}(t)\right), \\
L_{j(q-1)}^{2}(t)= & \sum_{\kappa=1}^{r} K_{j \kappa}\left(t, y_{q-1}(t)\right) x_{\kappa(q-1)}^{m}\left(y_{q-1}(t)\right),
\end{aligned}
$$

where

$$
\begin{array}{rlrl}
\Delta x_{i(q)}(t) & =x_{i(q)}(t)-x_{i(q-1)}(t), & & i=1, \ldots, r, \\
\Delta y_{q}(t) & =y_{q}(t)-y_{q-1}(t), & & \\
\Delta z_{j(q)}(t) & =z_{j(q)}(t)-z_{j(q-1)}(t), & & j=1, \ldots, p \\
& q=2,3, \ldots
\end{array}
$$

Remark: the first equation of system (18) is a linear Volterra integral equation of second kind, so it has a unique solution in terms of $\Delta x_{i(q)}(t)$, since $G(t)$ has no zeros on the interval $\left[t_{0}, T\right]$ and the kernels $\Omega_{i, j}(t, \tau), i, j=1,2, \ldots, r$ are continuous functions, that can be evaluated by the method of successive approximation, then the sequences $\Delta y_{q}(t)$ and $\Delta z_{j(q)}(t)$ can be uniquely determined from the second and third equations of the system (18).

\section{GAUSS-LEGENDRE QUADRATURE METHOD FOR APPROXIMATE SOLUTION}

For the approximate solution of system (18), we define a grid $(\omega)$ of points $t_{\alpha}=t_{0}+\alpha\left(T-t_{0}\right) / d$, $\alpha=0,1, \ldots, d$, where $d$ refers to the number of partitions in $\left[t_{0}, T\right]$. Hence we obtain the following system

$$
\begin{gathered}
\Delta x_{i(q)}\left(t_{\alpha}\right)+m \sum_{j=1}^{r} \int_{y_{0}\left(t_{\alpha}\right)}^{t_{\alpha}}\left(\Omega_{i j}\left(t_{\alpha}, \tau\right) x_{j 0}^{m-1}(\tau)\right. \\
\left.\Delta x_{j(q)}(\tau)\right) \mathrm{d} \tau=\Phi_{i(q-1)}\left(t_{\alpha}\right), \quad i=1, \ldots, r, \\
\Delta y_{q}\left(t_{\alpha}\right)=\frac{1}{G(t)}\left[m \sum _ { i = 1 } ^ { r } \int _ { y _ { 0 } ( t _ { \alpha } ) } ^ { t _ { \alpha } } \left[\sum _ { \kappa = 1 } ^ { r } \left(H_{\kappa i}\left(t_{\alpha}, \tau\right)\right.\right.\right. \\
\left.x_{i 0}^{m-1}(\tau) \Delta x_{i(q)}(\tau)\right)+\sum_{\kappa=1}^{p}\left(K_{\kappa i}\left(t_{\alpha}, \tau\right) x_{i 0}^{m-1}(\tau)\right. \\
\left.\left.\Delta x_{i(q)}(\tau)\right)\right] \mathrm{d} \tau+\sum_{i=1}^{r} F_{i(q-1)}^{1}\left(t_{\alpha}\right)+\sum_{j=1}^{p} F_{j(q-1)}^{2}\left(t_{\alpha}\right) \\
\left.-F_{q-1}^{3}\left(t_{\alpha}\right)\right],
\end{gathered}
$$




$$
\begin{aligned}
& \Delta z_{j(q)}\left(t_{\alpha}\right)=F_{j(q-1)}^{2}\left(t_{\alpha}\right) \\
& +m \sum_{\kappa=1}^{r} \int_{y_{0}\left(t_{\alpha}\right)}^{t_{\alpha}} K_{j \kappa}\left(t_{\alpha}, \tau\right) x_{\kappa 0}^{m-1}(\tau) \Delta x_{\kappa(q)}(\tau) \mathrm{d} \tau \\
& +\Delta y\left(t_{\alpha}\right)_{q} L_{j(q-1)}^{2}\left(t_{\alpha}\right), \quad j=1, \ldots, p, \\
& \quad \alpha=1, \ldots, d, \quad
\end{aligned}
$$

and

$$
\begin{gathered}
\Phi_{i(q-1)}\left(t_{\alpha}\right)=F_{i(q-1)}^{1}\left(t_{\alpha}\right) \\
+\frac{L_{i(q-1)}^{1}\left(t_{\alpha}\right)}{G\left(t_{\alpha}\right)}\left[F_{n-1}^{3}\left(t_{\alpha}\right)-\sum_{i=1}^{r} F_{i(q-1)}^{1}\left(t_{\alpha}\right)\right. \\
\left.+\sum_{j=1}^{p} F_{j(q-1)}^{2}\left(t_{\alpha}\right)\right], \\
F_{i(q-1)}^{1}\left(t_{\alpha}\right)=\int_{y_{q-1}\left(t_{\alpha}\right)}^{t_{\alpha}} \sum_{\kappa=1}^{r} H_{i \kappa}\left(t_{\alpha}, \tau\right) x_{\kappa(q-1)}^{m}(\tau) \mathrm{d} \tau \\
-x_{i(q-1)}\left(t_{\alpha}\right), \quad i=1, \ldots, r, \\
F_{j(q-1)}^{2}\left(t_{\alpha}\right)=\int_{y_{q-1}\left(t_{\alpha}\right)}^{t_{\alpha}} \sum_{\kappa=1}^{r} K_{j \kappa}\left(t_{\alpha}, \tau\right) x_{\kappa(q-1)}^{m}(\tau) \mathrm{d} \tau \\
-z_{i(q-1)}\left(t_{\alpha}\right), \quad j=1, \ldots, p, \\
F_{q-1}^{3}\left(t_{\alpha}\right)=c\left(t_{\alpha}\right)-\sum_{i=1}^{r} x_{i(q-1)}\left(t_{\alpha}\right)-\sum_{j=1}^{p} z_{j(q-1)}\left(t_{\alpha}\right), \\
L_{i(q-1)}^{1}\left(t_{\alpha}\right)=\sum_{\kappa=1}^{r}\left[H_{i \kappa}\left(t_{\alpha}, y_{q-1}(t)\right) x_{\kappa(q-1)}^{m}\right. \\
\left.\left(y_{q-1}\left(t_{\alpha}\right)\right)\right], \quad i=1, \ldots, r, \\
L_{j(q-1)}^{2}\left(t_{\alpha}\right)=\sum_{\kappa=1}^{r}\left[K_{j \kappa}\left(t, y_{q-1}\left(t_{\alpha}\right)\right) x_{\kappa(q-1)}^{m}\right. \\
\left.\left(y_{q-1}\left(t_{\alpha}\right)\right)\right], \quad j=1, \ldots, p .
\end{gathered}
$$

The powerful technique to approximate all integrations in the system (19) is Gauss-Legendre quadrature formula, so that for an arbitrary interval $[a, b]$ we have the following form

$$
\int_{a}^{b} f(t) \mathrm{d} t=\frac{b-a}{2} \sum_{j=1}^{d} w_{j} f\left(t_{j}\right)+R_{d},
$$

where the nodes $t_{j}=\left(\frac{1}{2}(b-a)\right) x_{j}+\frac{1}{2}(b+a)$ are related to the zeros $x_{j}$ of Legendre polynomial $P_{d}(x)$ over the interval $[-1,1]$, with $P_{d}(1)=1$ and the three term recurrence relation is

$$
(d+1) P_{d+1}(x)=(2 d+1) x P_{d}(x)-d P_{d-1}(x),
$$

and the weight function $w(t)=1$, where

$$
w_{j}=\frac{2}{\left(1-x_{j}^{2}\right)\left[P_{d}^{\prime}\left(x_{j}\right)\right]^{2}}, \quad j=1, \ldots, d
$$

and the remainder is

$$
R_{d}=\frac{2^{2 d+1}(d !)^{4}}{(2 d+1)[2 d !]^{3}} f^{2 d}(\xi),-1<\xi<1 .
$$

We introduce a subgrid $\left(\omega_{1}\right)$ of $\ell$ Legendre knot points at each subinterval $\left(y_{0}\left(t_{\alpha}\right), t_{\alpha}\right)$ such that

$$
\begin{aligned}
\tau_{\alpha}^{v}=\frac{t_{\alpha}-y_{0}\left(t_{\alpha}\right)}{2} x_{v}+\frac{t_{\alpha}+y_{0}\left(t_{\alpha}\right)}{2}, \\
v=1, \ldots, \ell, \quad \alpha=1, \ldots, d .
\end{aligned}
$$

$x_{v}$ represents the zeros of Legendre polynomial $P_{d}(x)$ over the interval $[-1,1]$ and $\tau_{\alpha}^{v} \neq t_{\alpha}$. Applying Gauss-Legendre quadrature formula (20) for the integral system (19) at the Legendre grid points $\tau_{\alpha}^{v}$, we obtain

$$
\begin{gathered}
\Delta x_{i(q)}\left(\tau_{\alpha}^{v}\right)+m \sum_{j=1}^{r}\left(\frac{t_{\alpha}-y_{0}\left(t_{\alpha}\right)}{2}\right) \\
\sum_{\kappa=1}^{\ell} \Omega_{i j}\left(\tau_{\alpha}^{v}, \tau_{\alpha}^{\kappa}\right) x_{j 0}^{m-1}\left(\tau_{\alpha}^{\kappa}\right) \Delta x_{j(q)}\left(\tau_{\alpha}^{\kappa}\right) w_{\kappa} \\
=\Phi_{i(q-1)}\left(\tau_{\alpha}^{v}\right), \quad i=1, \ldots, r, \\
\Delta y_{q}\left(\tau_{\alpha}^{v}\right)=\frac{1}{G(t)}\left[m \sum_{i=1}^{r}\left(\frac{t_{\alpha}-y_{0}\left(t_{\alpha}\right)}{2}\right)\right. \\
\sum_{j=1}^{\ell}\left[\sum_{\kappa=1}^{r} H_{\kappa i}\left(\tau_{\alpha}^{v}, \tau_{\alpha}^{j}\right) x_{i 0}^{m-1}\left(\tau_{\alpha}^{j}\right) \Delta x_{i(q)}\left(\tau_{\alpha}^{j}\right)\right. \\
\left.+\sum_{\kappa=1}^{p} K_{\kappa i}\left(\tau_{\alpha}^{v}, \tau_{\alpha}^{j}\right) x_{i 0}^{m-1}\left(\tau_{\alpha}^{j}\right) \Delta x_{i(q)}\left(\tau_{\alpha}^{j}\right)\right] w_{j} \\
\left.+\sum_{i=1}^{r} F_{i(q-1)}^{1}\left(\tau_{\alpha}^{v}\right)+\sum_{j=1}^{p} F_{j(q-1)}^{2}\left(\tau_{\alpha}^{v}\right)-F_{q-1}^{3}\left(\tau_{\alpha}^{v}\right)\right], \\
\Delta z_{j(q)}\left(\tau_{\alpha}^{v}\right)=F_{j(q-1)}^{2}\left(\tau_{\alpha}^{v}\right) \\
+m \sum_{\kappa=1}^{r}\left(\frac{t_{\alpha}-y_{0}\left(t_{\alpha}\right)}{2}\right) \sum_{i=1}^{\ell} K_{j \kappa}\left(\tau_{\alpha}^{v}, \tau_{\alpha}^{i}\right) \\
x_{\kappa 0}^{m-1}(\tau) \Delta x_{\kappa(q)}\left(\tau_{\alpha}^{i}\right) w_{i}+\Delta y\left(\tau_{\alpha}^{v}\right)_{q} L_{j(q-1)}^{2}\left(\tau_{\alpha}^{v}\right), \\
j=1, \ldots, p, \quad v=1, \ldots, \ell, \alpha=1, \ldots, d, \quad(24),
\end{gathered}
$$

where

$$
\begin{aligned}
\Phi_{i(q-1)}\left(\tau_{\alpha}^{v}\right)=F_{i(q-1)}^{1}\left(\tau_{\alpha}^{v}\right)+\frac{L_{i(q-1)}^{1}\left(\tau_{\alpha}^{v}\right)}{G\left(\tau_{\alpha}^{v}\right)} \\
{\left[F_{q-1}^{3}\left(\tau_{\alpha}^{v}\right)-\sum_{i=1}^{r} F_{i(q-1)}^{1}\left(\tau_{\alpha}^{v}\right)+\sum_{j=1}^{p} F_{j(q-1)}^{2}\left(\tau_{\alpha}^{v}\right)\right], }
\end{aligned}
$$




$$
\begin{gathered}
F_{i(q-1)}^{1}\left(\tau_{\alpha}^{v}\right)=\left(\frac{t_{\alpha}-y_{q-1}\left(t_{\alpha}\right)}{2}\right) \\
\sum_{j=1}^{\ell} \sum_{\kappa=1}^{r} H_{i \kappa}\left(\tau_{\alpha}^{v}, \tau_{\alpha}^{j}\right) x_{\kappa(q-1)}^{m}\left(\tau_{\alpha}^{j}\right) w_{j} \\
-x_{i(q-1)}\left(\tau_{\alpha}^{v}\right), \quad i=1, \ldots, r, \\
F_{j(q-1)}^{2}\left(\tau_{\alpha}^{v}\right)=\left(\frac{t_{\alpha}-y_{q-1}\left(t_{\alpha}\right)}{2}\right) \\
\sum_{i=1}^{\ell} \sum_{\kappa=1}^{r} K_{j \kappa}\left(\tau_{\alpha}^{v}, \tau_{\alpha}^{i}\right) x_{\kappa(q-1)}^{m}\left(\tau_{\alpha}^{i}\right) w_{i} \\
-z_{i(q-1)}\left(\tau_{\alpha}^{v}\right), \quad j=1, \ldots, p, \\
F_{q-1}^{3}\left(\tau_{\alpha}^{v}\right)=c\left(\tau_{\alpha}^{v}\right)-\sum_{i=1}^{r} x_{i(q-1)}\left(\tau_{\alpha}^{v}\right)-\sum_{j=1}^{p} z_{j(q-1)}\left(\tau_{\alpha}^{v}\right), \\
L_{i(q-1)}^{1}\left(\tau_{\alpha}^{v}\right)=\sum_{\kappa=1}^{r} H_{i \kappa}\left(\tau_{\alpha}^{v}, y_{q-1}\left(\tau_{\alpha}^{v}\right)\right) x_{\kappa(q-1)}^{m} \\
\left(y_{q-1}\left(\tau_{\alpha}^{v}\right)\right), \quad i=1, \ldots, r, \\
L_{j(q-1)}^{2}\left(\tau_{\alpha}^{v}\right)=\sum_{\kappa=1}^{r} K_{j \kappa}\left(\tau_{\alpha}^{v}, y_{q-1}\left(\tau_{\alpha}^{v}\right)\right) \\
x_{\kappa(q-1)}^{m}\left(y_{q-1}\left(\tau_{\alpha}^{v}\right)\right), \quad j=1, \ldots, p,
\end{gathered}
$$

where $v=1, \ldots, \ell, \alpha=1, \ldots, d$. The first equation of the system (24) is a linear algebraic system of $d \times l$ equations and $d \times l$ unknowns. If the matrix of this system is not singular then it has a unique solution in terms of $\Delta x_{i(q)}\left(\tau_{\alpha}^{v}\right), i=1, \ldots, r, s=2,3, \ldots$, $v=1, \ldots, \ell, \alpha=1, \ldots, d$, then the values of $\Delta y_{q}\left(\tau_{\alpha}^{v}\right)$ and $\Delta z_{j(q)}\left(\tau_{\alpha}^{v}\right), j=1, \ldots, p$ can be easily evaluated by direct substitution the value of $\Delta x_{i(q)}\left(\tau_{\alpha}^{v}\right)$ in the second equation of system (24) and $\Delta x_{i(q)}\left(\tau_{\alpha}^{v}\right)$ and $\Delta y_{q}\left(\tau_{\alpha}^{v}\right)$ in the third equations, respectively. Since the values of the functions $x_{i(q)}\left(\tau_{\alpha}^{v}\right)$ are known at $l$ Legendre grid points in each subinterval $\left(y_{0}\left(t_{\alpha}\right), t_{\alpha}\right)$ for each $q$ iteration, the values of unknown functions $x_{i}\left(t_{\alpha}\right)$ can be found by applying the Newton forward interpolation formula which are

$$
\begin{aligned}
x_{i(q)}(t) \simeq & P_{\ell}(t) \\
= & x_{i(q)}\left(\tau_{\alpha}^{\ell}\right)+x_{i(q)}\left(\tau_{\alpha}^{\ell}, \tau_{\alpha}^{\ell-1}\right)\left(t-\tau_{\alpha}^{\ell}\right) \\
& +x_{i(q)}\left(\tau_{\alpha}^{\ell}, \tau_{\alpha}^{\ell-1}, \tau_{\alpha}^{\ell-2}\right)\left(t-\tau_{\alpha}^{\ell}\right)\left(t-\tau_{\alpha}^{\ell-1}\right) \\
& +\cdots+x_{i(q)}\left(\tau_{\alpha}^{\ell}, \tau_{\alpha}^{\ell-1}, \tau_{\alpha}^{\ell-2}, \ldots, \tau_{\alpha}^{1}\right) \\
& \quad \times\left(t-\tau_{\alpha}^{\ell}\right)\left(t-\tau_{\alpha}^{\ell-1}\right) \cdots\left(t-\tau_{\alpha}^{1}\right) .
\end{aligned}
$$

It is known ${ }^{9}$ that the error of (25) is

$$
\left|x_{i(q)}(t)-P_{\ell}(t)\right| \leqslant \frac{M}{\ell+1 !},
$$

Table 1 Numerical results for (26) with $d=10, h=0.5$, $\ell=5$.

\begin{tabular}{cccc}
\hline$q$ & $\epsilon_{x}$ & $\epsilon_{z}$ & $\epsilon_{y}$ \\
\hline 1 & 0.00 & $1.014137 \times 10^{-4}$ & 0.022024 \\
2 & 0.00 & $6.931068 \times 10^{-5}$ & 0.015093 \\
5 & 0.00 & $2.228037 \times 10^{-5}$ & 0.004871 \\
10 & 0.00 & $3.416982 \times 10^{-6}$ & $8.201659 \times 10^{-4}$ \\
20 & 0.00 & $1.190459 \times 10^{-7}$ & $3.166210 \times 10^{-5}$ \\
40 & 0.00 & $2.350333 \times 10^{-10}$ & $6.639312 \times 10^{-8}$ \\
\hline
\end{tabular}

Table 2 Numerical results for (26) with $d=10, h=0.5$, $\ell=10$.

\begin{tabular}{cccc}
\hline$q$ & $\epsilon_{x}$ & $\epsilon_{z}$ & $\epsilon_{y}$ \\
\hline 1 & 0.00 & $7.615760 \times 10^{-5}$ & 0.00167 \\
2 & 0.00 & $5.112875 \times 10^{-7}$ & $8.618341 \times 10^{-4}$ \\
5 & 0.00 & $4.964108 \times 10^{-9}$ & $2.761149 \times 10^{-5}$ \\
10 & 0.00 & $4.287655 \times 10^{-11}$ & $9.764381 \times 10^{-7}$ \\
20 & 0.00 & $1.005649 \times 10^{-12}$ & $3.914765 \times 10^{-9}$ \\
40 & 0.00 & $3.964122 \times 10^{-15}$ & $4.765199 \times 10^{-13}$ \\
\hline
\end{tabular}

where

$$
M=\max \left\{\left|x_{i(q)}^{\ell+1}(\xi) \cdot\left(t-\tau_{\alpha}^{1}\right) \cdots\left(t-\tau_{\alpha}^{\ell}\right)\right|\right\} .
$$

From the system (24) it follows that by increasing the knot points $l$ the more accurate solution is obtained, therefore the Newton forward interpolation method can be used for reasonable amount of $l$.

\section{NUMERICAL EXAMPLES}

Consider the system of nonlinear Volterra integral equation

$$
x(t)=\int_{y(t)}^{t} t x^{2}(\tau) \mathrm{d} \tau, \quad z(t)=\int_{y(t)}^{t} t x^{2}(\tau) \mathrm{d} \tau,
$$

and $c(t)=2 t$, where $t \in\left[t_{0}, T\right]=[5,10]$, and the exact solution of (26) is $x^{*}(t)=t, z^{*}(t)=t$, and $y^{*}(t)=\sqrt[3]{t^{3}-3}$. In this particular example, initial guesses are $x_{0}(t)=2 t, z_{0}(t)=\frac{1}{2} t$, and $y_{0}(t)=$ $\sqrt[5]{t^{5}-5}$.

Tables $1,2,3$, and 4 show that $x_{q}(t)$ coincides with the exact $x^{*}(t)$ from the first iteration, whereas $z_{q}(t)$ and $y_{q}(t)$ are close to $z^{*}(t)$ and $y^{*}(t)$, respectively, after some iterations. Notation used here are $d$ is the number of nodes, $l$ is the number of subnodes, $q$ is the number of iterations, and

$$
\begin{aligned}
\epsilon_{x} & =\max _{t \in[5,10]}\left|x_{q}(t)-x^{*}(t)\right|, \\
\epsilon_{z} & =\max _{t \in[5,10]}\left|z_{q}(t)-z^{*}(t)\right|,
\end{aligned}
$$


Table 3 Numerical results for (26) with $d=20, h=0.25$, $\ell=5$.

\begin{tabular}{cccc}
\hline$q$ & $\epsilon_{x}$ & $\epsilon_{z}$ & $\epsilon_{y}$ \\
\hline 1 & 0.00 & $1.013038 \times 10^{-4}$ & 0.020035 \\
2 & 0.00 & $6.032177 \times 10^{-5}$ & 0.009872 \\
5 & 0.00 & $1.997625 \times 10^{-5}$ & 0.002792 \\
10 & 0.00 & $3.223779 \times 10^{-6}$ & $8.170566 \times 10^{-4}$ \\
20 & 0.00 & $1.084199 \times 10^{-7}$ & $3.009728 \times 10^{-5}$ \\
40 & 0.00 & $2.197287 \times 10^{-10}$ & $6.290796 \times 10^{-8}$ \\
\hline
\end{tabular}

Table 4 Numerical results for (26) with $d=20, h=0.25$, $\ell=10$.

\begin{tabular}{cccc}
\hline$q$ & $\epsilon_{x}$ & $\epsilon_{z}$ & $\epsilon_{y}$ \\
\hline 1 & 0.00 & $7.291381 \times 10^{-5}$ & 0.00097 \\
2 & 0.00 & $5.001363 \times 10^{-7}$ & $8.2875310 \times 10^{-4}$ \\
5 & 0.00 & $4.103774 \times 10^{-9}$ & $2.4910043 \times 10^{-5}$ \\
10 & 0.00 & $4.011820 \times 10^{-11}$ & $9.3871220 \times 10^{-7}$ \\
20 & 0.00 & $9.003781 \times 10^{-13}$ & $3.6992101 \times 10^{-9}$ \\
40 & 0.00 & $3.210009 \times 10^{-15}$ & $4.4000439 \times 10^{-13}$ \\
\hline
\end{tabular}

$$
\epsilon_{y}=\max _{t \in[5,10]}\left|y_{q}(t)-y^{*}(t)\right| .
$$

\section{CONCLUSIONS}

In this article, the approximate solution of $n \times n$ system of nonlinear Volterra integral equations by the Newton-Kantorovich method is discussed and the uniqueness of the solution is shown. A new interesting idea has been proposed by introducing a subgrid of collocation points $\tau_{\alpha}^{v}, \alpha=1,2, \ldots, d$, $v=1,2, \ldots, l$ which are included in $y_{0}\left(t_{\alpha}, t_{\alpha}\right]$. To obtain a good accuracy it is enough to increase $\ell$ but not $d$ as shown in Tables 1, 2, 3, and 4. This is the advantage of the present approach of solving the $n \times n$ system of nonlinear Volterra integral equations.

Acknowledgements: This work was supported by Universiti Putra Malaysia under the Research Grand Universiti Putra Malaysis (Geran Putra, 2015). Project code is $G P-i(2014) 9442300$. Authors are grateful for financial support for the Research Management Centre, Universiti Putra Malaysia.

\section{REFERENCES}

1. Goghary H, Javadi Sh Babolian E (2005) Restarted Adomian method for system of nonlinear Volterra integral equations. Appl Math Comput 161, 745-51.

2. Dobriţoiu M, Şerban MA (2014) Step method for a system of integral equations from biomathematics. Appl Math Comput 227, 412-21.

3. Yang LH, Li HY, Wang JR (2013) Solving a system of linear Volterra integral equations using the modified reproducing kernel method. Abstr Appl Anal 2013, ID 196308.

4. Biazar J, Ebrahimi H (2012) Chebyshev wavelets approach for nonlinear systems of Volterra integral equations. Comput Math Appl 63, 608-16.

5. Berenguer MI, Gámez D, Garralda-Guillem AI, Ruiz Galán M, Serrano Pérez MC (2011) Biorthogonal systems for solving Volterra integral equation systems of the second kind. $J$ Comput Appl Math 235, 1875-83.

6. Balakumar V, Murugesan K (2014) Single-Term Walsh Series method for systems of linear Volterra integral equations of the second kind. Appl Math Comput 228, 371-6.

7. Vladimirovich B, Tynda A (2003) Approximate solution of nonlinear integral equations of the theory of developing systems. Differ Equat 39, 1277-88.

8. Kantorovich L, Akilov G (1982) Functional Analysis, Pergamon Press, Oxford.

9. Atkinson KE (1997) The Numerical Solution of Integral Equations of the Second Kind, Cambridge Univ Press. 